\title{
Polymorphism and its influence on metathesis reactions
}

\author{
Rebecca D. McAuliffe ${ }^{\mathrm{a}}$, Paul K. Todd ${ }^{\mathrm{b}}$, James R. Neilson ${ }^{\mathrm{c}}$, and Gabriel M. Veith ${ }^{\mathrm{d}}$ \\ a'Oak Ridge National Laboratory, 1 Bethel Valley Road, Oak Ridge, TN 37830, mcaulifferd@ornl.gov \\ bColorado State University, 1872 Campus Delivery, Fort Collins, CO 80523, paul.todd@colostate.edu \\ ${ }^{\mathrm{c} C}$ Colorado State University, 1872 Campus Delivery, Fort Collins, CO 80523, \\ james.neilson@,colostate.edu \\ dOak Ridge National Laboratory, 1 Bethel Valley Road, Oak Ridge, TN 37830, veithgm@ornl.gov
}

Three different polymorphs of $\mathrm{LiMnO}_{2}$ can be used as precursors to synthesize $\mathrm{YMnO}_{\mathrm{x}}$ via metathesis reactions. This talk investigates how metathesis reaction pathways are affected by different precursor structures. While it has been shown that the alkali precursor can have a large effect on the reaction pathway and the polymorph of the oxide formed, the effects of initial precursor structure is not well understood. ${ }^{1}$ In situ X-ray and neutron diffraction studies were performed to investigate the formation of intermediate phases and reaction kinetics for all three $\mathrm{LiMnO}_{2}$ polymorphs. By utilizing in situ neutron diffraction, the movement of $\mathrm{Li}$ and $\mathrm{O}$ can be tracked throughout structures during the reaction process. This study provides an understanding of the reaction mechanisms in metathesis reactions and highlights the importance of using in situ techniques to better understand the reaction pathways in the syntheses of complex oxides.

This work was supported as part of GENESIS: A Next Generation Synthesis Center, an Energy Frontier Research Center funded by the U.S. Department of Energy, Office of Science, Basic Energy Sciences under Award Number DE-SC0019212

\section{$\underline{\text { References }}$}

[1] Todd, P. K. \& Neilson, J. R. Selective Formation of Yttrium Manganese Oxides through Kinetically Competent Assisted Metathesis Reactions. (2019) J. Am. Chem. Soc. 141, 1191-1195. 